\title{
Pretreatment Advanced Imaging in Patients with Stroke Treated with IV Thrombolysis: Evaluation of a Multihospital Data Base
}

\author{
J.S. McDonald, J. Fan, D.F. Kallmes, and H.J. Cloft
}

\begin{abstract}
BACKGROUND AND PURPOSE: CT angiography, CT perfusion, and MR imaging have all been advocated as potentially useful in treatment planning for patients with acute ischemic stroke. We evaluated a large multihospital data base to determine how the use of advanced imaging is evolving in patients treated with intravenous thrombolysis.
\end{abstract}

MATERIALS AND METHODS: Patients with acute ischemic stroke receiving IV thrombolytic therapy from 2008 to 2011 were identified by using the Premier Perspective data base. Mortality and discharge to long-term care rates were compared following multivariate logistic regression between patients who received head CT only versus those who received CTA without CT perfusion, CT perfusion, or MR imaging.

RESULTS: Of 12,429 included patients, 7305 (59\%) were in the CT group, 2359 (19\%) were in the CTA group, 848 (7\%) were in the CTP group, and 1917 (15\%) were in the MR group. From 2008 to 2011, the percentage of patients receiving head CT only decreased from 64\% to 55\%, while the percentage who received cerebral CT perfusion increased from $3 \%$ to $8 \%$. The use of CT angiography and MR imaging marginally increased (1\%-2\%). Outcomes were similar between CT only and advanced imaging patients, except discharge to long-term care was slightly more frequent in the CTP group (OR $=1.17[95 \% \mathrm{Cl}, 0.96-1.43] ; P=.0412)$ and $\mathrm{MR}$ group $(\mathrm{OR}=1.14[95 \% \mathrm{Cl}, 1.01-1.28] ; P=.0177)$ and mortality was lower in the MR group $(\mathrm{OR}=0.64[95 \% \mathrm{Cl}, 0.52-0.79] ; P<.0001)$.

CONCLUSIONS: Use of advanced imaging is increasing in patients treated with IV thrombolysis. While there were differences in outcomes among imaging groups, the clinical effect of advanced imaging remains unclear.

$\mathrm{T}$ he potential benefit of intravenous thrombolytic therapy for acute ischemic stroke decreases rapidly with time. Because of wide variability in collateral circulation, time elapsed since onset is a crude indicator of the potential benefit of treatment in each patient. Advanced imaging might provide a means to refine selection of patients who could potentially benefit from revascularization therapy. The advanced imaging techniques that might add clinically useful information in the setting of acute ischemic stroke include CT angiography, CT perfusion, and MR imaging. CT angiography can be used to identify patients with large-artery occlusions potentially amenable to intra-arterial therapy, ${ }^{1-3}$ and CT angiography source images have been proposed as a means of

Received June 11, 2013; accepted after revision July 3.

From the Departments of Radiology (J.S.M., D.F.K., H.J.C.), Health Sciences Research (J.F.), and Neurosurgery (D.F.K., H.J.C.), Mayo Clinic, Rochester, Minnesota.

Please address correspondence to Jennifer S. McDonald, PhD, Department of Radiology, Mayo Clinic, 200 1st St SW, Rochester, MN 55905; e-mail: mcdonald. jennifer@mayo.edu

三Indicates article with supplemental on-line table.

http://dx.doi.org/10.3174/ajnr.A3797 evaluating collateral circulation. ${ }^{4-6} \mathrm{CT}$ perfusion may potentially allow discrimination between salvageable brain ("penumbra") and brain already doomed to infarction ("ischemic core") ) $^{2,7-10}$ and thus may be useful in helping to refine selection of patients for IV thrombolysis ${ }^{11,12}$ or intra-arterial thrombectomy. ${ }^{13-15} \mathrm{MR}$ perfusion and diffusion imaging have also been reported to be useful in screening patients for intravenous therapy. ${ }^{16-22}$

While advanced imaging techniques hold promise for the evaluation of patients with acute ischemic stroke, there is variation in techniques and definitions of parameters that limit wide application and acceptance of these techniques. ${ }^{4,23-25}$ There is currently no consensus on a standard imaging approach for acute ischemic stroke. We studied a large data base of hospitals in the United States to assess the recent use of advanced imaging in patients with acute ischemic stroke treated with intravenous thrombolysis, including an evaluation of the use of advanced imaging, with respect to patient outcome.

\section{MATERIALS AND METHODS}

Data Source and Study Population

The Perspective data base is a voluntary, fee-supported collection of data developed by Premier Inc (Charlotte, North Carolina) to 
assess quality and resource use. As of 2011, the Perspective data base consisted of approximately $15 \%$ of hospitalizations nationwide and represented $>600$ US hospitals. Detailed information of a patient's hospitalization, including patient demographics, hospital information, diagnoses, procedures, discharge status, payer, and all billed items are recorded.

Patients who presented with acute ischemic stroke (ICD9-CM diagnostic codes 433.x1 and 434.x1) from 2008 through 2011 were identified from the Perspective data base. Patients were included only if the stroke code was listed as the primary hospitalization diagnosis to avoid including patients with a history of stroke. Patients in this cohort who received IV thrombolysis during their hospitalization were identified by using the ICD-9 procedural code 99.10 .

Relevant imaging performed during hospitalization was retrieved by using billing information. Imaging was included if it was performed before or on the day of thrombolysis. Patients were included if they underwent a noncontrast head CT during hospitalization. Advanced imaging modalities of head CT angiography, cerebral CT perfusion, head MR imaging, and head MR angiography were also retrieved.

\section{Outcome Analysis}

The outcomes examined in this study were in-hospital mortality and discharge to long-term care (discharge to a rehabilitation facility, long-term care hospital, or nursing facility). Patients were divided into the following subgroups on the basis of imaging received: noncontrast head CT only (CT group), CT with cerebral CT angiography but no CT perfusion (CTA group), CT with cerebral CT perfusion (CTP group), and CT with head MR imaging or MR angiography (MR group). Statistical analyses were performed by using JMP, Version 9, and SAS, Version 9.3 (SAS Institute, Cary, North Carolina). Differences among imaging groups and admit years were compared by using the Pearson $\chi^{2}$ test and linear regression, respectively. The likelihood of mortality or discharge to long-term care was compared among imaging subgroups following multivariate logistic regression to minimize differences in clinical characteristics among groups. The generalized estimating equation was used to adjust for the possible clustering effect of hospitals in the patient population. ${ }^{26}$ Models were estimated by using the SAS GENMOD procedure (http://www. math.wpi.edu/saspdf/stat/chap29.pdf). Two models were created, a full model that encompassed all patient and hospital characteristics as shown in On-line Table 1 and a reduced model that encompassed only significant characteristics as determined by the stepwise selection option in the SAS LOGISTIC procedure (http://support.sas.com/documentation/cdl/en/statug/63347/ HTML/default/viewer.htm\#logistic_toc.htm).

\section{RESULTS}

Of the 176,991 patients identified who were hospitalized from 2008 to 2011 with acute ischemic stroke listed as a primary diagnostic code, 15,470 patients $(8.7 \%)$ received IV thrombolytic therapy during hospitalization. Of that group, 12,429 patients $(80 \%)$ underwent a head CT during hospitalization. Within that group, 2359 patients (19\%) also underwent a head CT angiography; 848 patients $(6.8 \%)$, cerebral CT perfusion; and 1917 pa-

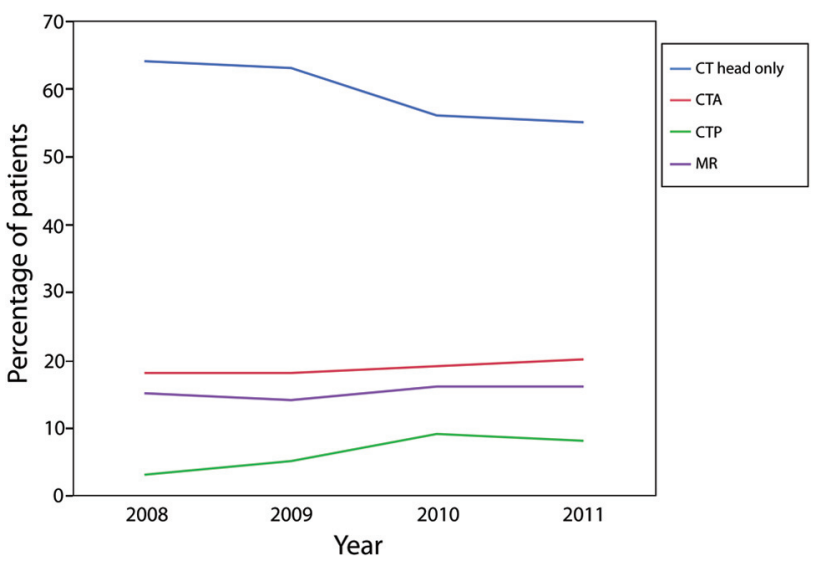

FIG 1. Trends in the use of head CT and advanced imaging in patients treated with IV thrombolysis from 2008 to 2011. Patients who received only head CT (blue line), head CT with CT angiography (red line), head $\mathrm{CT}$ with CT perfusion (green line), or head CT with MR imaging (purple line) are shown.

tients (15\%), MR imaging. Patient and hospital characteristics of the 4 imaging subgroups are shown in On-line Table 1.

Trends in the use of head CT and advanced imaging are shown in Fig 1. From 2008 to 2011, the percentage of patients treated with thrombolysis who only underwent a noncontrast head CT decreased slightly from $64 \%$ to $55 \%(P=.0570)$, while the percentage of patients who underwent cerebral CT perfusion increased from $3 \%$ to $8 \%(P=.11)$. Use of CT angiography and MR imaging each increased slightly from $18 \%$ to $20 \%(P=.0561)$ and from $15 \%$ to $16 \%(P=.33)$, respectively, during this time.

The incidence of in-hospital mortality was lower in the MR group $(6.2 \%)$ than in the CT, CTA, and CTP groups $(10.2 \%$, $12.4 \%$, and $12.4 \%$, respectively; $P<.0001$ ) (Table 1 ). The incidence of discharge to long-term care was slightly higher in the CTP group (47\%) compared with the CT, CTA, and MR groups (43\%, $41 \%$, and $43 \%$, respectively; $P=.0352$ ). Following multivariate logistic regression by using the full model, the likelihood of mortality was significantly lower in the MR group compared with the CT group (OR $=0.64$ [95\% CI, 0.52-0.79]; $P<.0001)$ but was similar between the CTA and CT groups $(P=.99)$ and between the CTP and CT groups $(P=.20)$ (Table 2). The likelihood of discharge to long-term care was significantly higher in the MR group compared with the CT group (OR $=1.14$ [95\% CI, 1.011.28]; $P=.0177$ ), was slightly higher in the CTP group compared with the CT group $(\mathrm{OR}=1.17$ [95\% CI, 0.96-1.43]; $P=.0412)$, and was similar between the CT and CTA groups $(P=.31)$. Similar findings were observed by using the reduced regression models.

\section{DISCUSSION}

Our analysis of a large, multihospital data base found wide variation in the use of imaging in patients with acute ischemic stroke. From 2008 to 2011, the percentage of patients treated with thrombolysis who received only a head CT decreased slightly from $64 \%$ to $55 \%$. If this trend continues, more than half of the patients treated with thrombolysis will receive some form of advanced imaging during their hospitalization. This growth in the use of 
Table 1: Outcome incidences by imaging subgroup

\begin{tabular}{lcccc}
\hline & CT Group & CTA Group & CTP Group & MR Group \\
\hline In-hospital mortality & $748 / 7305(10.2 \%)$ & $241 / 2359(12.4 \%)$ & $105 / 848(12.4 \%)$ & $119 / 1917(6.2 \%)$ \\
Discharge to long-term care & $3170 / 7305(43 \%)$ & $975 / 2359(41 \%)$ & $399 / 848(47 \%)$ & $828 / 1917(43 \%)$ \\
\hline
\end{tabular}

Table 2: Patient outcomes following logistic regression analysis

\begin{tabular}{lcc}
\hline & Odds Ratio (95\% CI) & $P$ Value \\
\hline In-hospital mortality & & \\
Full model & 1.00 (reference) & - \\
CT & $1.00(0.83-1.21)$ & .99 \\
CTA & $1.17(0.93-1.47)$ & .20 \\
CTP & $0.64(0.52-0.79)$ & $<.0001$ \\
MRI & 1.00 (reference) & \\
Reduced model & $1.03(0.86-1.24)$ & .75 \\
CT & $1.14(0.92-1.40)$ & .22 \\
CTA & $0.65(0.53-0.79)$ & $<.0001$ \\
CTP & & \\
MRI & & \\
Discharge to long-term care & $1.00($ reference) & - \\
Full model & $0.95(0.83-1.09)$ & .31 \\
CT & $1.17(0.96-1.43)$ & .0412 \\
CTA & $1.14(1.01-1.28)$ & .0177 \\
CTP & & \\
MRI & $1.00($ reference) & - \\
Reduced model & $0.89(0.66-1.18)$ & .59 \\
CT & $1.33(1.00-1.77)$ & .12 \\
CTA & $1.15(0.73-1.83)$ & .0471 \\
CTP &
\end{tabular}

Note:- - indicates no $P$ value was calculated for the CT reference group.

advanced imaging is primarily attributable to increased use of CT perfusion but also to increased use of CT angiography and MR imaging.

Imaging has been an essential part of stroke treatment planning since the introduction of CT. ${ }^{27}$ Unenhanced CT has been useful for demonstrating acute hemorrhage or other mimicking lesions, as well as showing cytotoxic edema from evolving infarction. CT is standard for trauma patients in emergency departments and has been the historical standard for patients with acute neurologic conditions, so CT is already fully integrated into emergency department culture and is readily available to patients with acute ischemic stroke. In fact, readily available head CT is a defining characteristic of a primary stroke center in the United States. ${ }^{28}$ If the patient is already going to the CT scanner for the standard unenhanced imaging, it is generally not very time-consuming to add additional CT angiography and/or CT perfusion studies. ${ }^{2}$ However, advanced CT, including high-quality CT perfusion and CT angiography, requires hardware and software that are not available at every hospital treating patients with stroke; this circumstance might limit adoption of these imaging techniques. As CT scanners are replaced and upgraded with time, advanced CT techniques are becoming uniformly available; this availability might account for some of the increased use demonstrated in our study. The clinical utility of such advanced CT imaging, however, is not universally accepted.

Our study showed that from 2008 to 2011 , screening with MR imaging had a negligible increase from $15 \%$ to $16 \%$ of patients undergoing IV thrombolysis. These results suggest that MR imaging has not been widely adopted. MR imaging has been proposed as an effective means of identifying candidates for intrave- nous thrombolysis in the 3- to 4.5 -hour time window. ${ }^{16-22}$ The exact time of onset relative to the time of imaging or the time of thrombolytic administration is not available in the Perspective data base, so we cannot determine how often MR imaging was used for screening patients in the 3- to 4.5-hour time window. In addition, MR imaging is not typically as readily available as CT in an emergency setting. Safety screening for implants can slow down the process of acquiring MR imaging, especially if it is not possible to immediately obtain an accurate and complete history, which occurs reasonably often in the setting of acute ischemic stroke. Another impediment is that rapid, emergent acquisition of MR imaging is not part of the culture at many hospital emergency departments.

We found that patients who underwent MR imaging had lower in-hospital mortality rates compared with patients who underwent a head CT only or other types of advanced imaging. However, patients who received MR imaging or CT perfusion had a higher rate of being discharged to long-term care compared with the head CT group. These findings suggest that the use of advanced imaging may affect these patient outcomes; however, a causal link cannot be confirmed from our observational study. It is likely that patients treated with IV thrombolysis who underwent imaging with only unenhanced CT were clinically different from those who underwent additional imaging. Patients who underwent only CT were more likely to be admitted from the emergency department compared with patients who also underwent advanced imaging. While we performed multivariate logistic regression to minimize these differences in clinical characteristics between imaging groups, differences in unmeasured clinical variables may affect our results. For example, patients treated with IV thrombolysis who underwent additional imaging were probably more likely to have severe stroke compared with patients who only underwent CT imaging, with the additional imaging used to help determine whether mechanical embolectomy was warranted. We cannot quantify such a trend because we cannot assess stroke severity at presentation.

Our study of a national cohort of inpatients who received IV thrombolysis did not show that the use of CT angiography or CT perfusion significantly improved patient outcomes. However, these findings do not suggest that these imaging examinations are not useful in specific clinical settings, such as studies comparing IV thrombolysis with combined IV and intra-arterial treatments. In such studies, CT angiography may be useful in both identifying and characterizing pretreatment arterial occlusive lesions and in identifying post treatment recanalization effects.

This study has several additional limitations. We acknowledge that coding inaccuracies occur, which can affect a retrospective study of any administrative data base; however, it is unlikely that such inaccuracies would be more prevalent in one imaging group over another. As noted above, we cannot assess stroke severity at the time of presentation. We are unable to identify patients with similar stroke severity who did not receive intravenous thrombol- 
ysis, so we do not have a control group to allow assessment of the benefit from different treatments. We cannot determine whether screening imaging was used uniformly at each center and thus was not subject to selection bias. We also are unable to assess the time of presentation, but the patients undergoing intravenous thrombolysis would typically be expected to have presented early enough for initiation of intravenous therapy within 3 hours of symptom onset. However, some patients may have been evaluated with imaging specifically for broadening of the time window to 4.5 hours, especially with MR imaging. ${ }^{16-22}$ Our study focused on the use of advanced imaging before or on the day of thrombolysis; the use of advanced imaging after treatment was not examined. Additional studies are needed to examine the use of advanced imaging in patients treated with IV thrombolysis and to examine the effect of this imaging on patient outcomes.

\section{CONCLUSIONS}

Significant variation occurs in imaging of patients with acute ischemic stroke treated with intravenous thrombolysis in the United States. The use of advanced imaging is increasing in these patients. While there were differences in outcome among the imaging groups, the clinical effect of advanced imaging remains unclear.

Disclosures: Jennifer S. McDonald-UNRELATED: Grants/Grants Pending: GE Healthcare, ${ }^{*}$ Comments: unrelated investigator-initiated research grant. David F. Kallmes_UNRELATED: Consultancy: Codman, ${ }^{*}$ ev3, ${ }^{*}$ Medtronic, ${ }^{*}$ Comments: planning and implementation of clinical trials, Grants/Grants Pending: MicroVention,* Codman, ${ }^{*}$ ev3, Sequent, ${ }^{*}$ Surmodics, ${ }^{*}$ Comments: preclinical and clinical studies, Royalties: University of Virginia Patent Foundation, Comments: spine fusion. Harry J. Cloft_UNRELATED: Grants/Grants Pending: Cordis Endovascular, ${ }^{*}$ Comments: site principal investigator at enrolling site for Stenting and Angioplasty with Protection in Patients and HIgh Risk for Endarterectomy registry sponsored by Cordis Endovascular. *Money paid to the institution.

\section{REFERENCES}

1. Sylaja PN, Puetz V, Dzialowski I, et al. Prognostic value of CT angiography in patients with suspected vertebrobasilar ischemia. J Neuroimaging 2008;18:46-49

2. de Lucas EM, Sanchez E, Gutierrez A, et al. CT protocol for acute stroke: tips and tricks for general radiologists. Radiographics 2008;28:1673-87

3. Frölich AM, Psychogios MN, Klotz E, et al. Angiographic reconstructions from whole-brain perfusion CT for the detection of large vessel occlusion in acute stroke. Stroke 2012;43:97-102

4. Pulli B, Schaefer PW, Hakimelahi R, et al. Acute ischemic stroke: infarct core estimation on CT angiography source images depends on CT angiography protocol. Radiology 2012;262:593-604

5. Pulli B, Yoo AJ. CT angiography source images with modern multisection CT scanners: delay time from contrast injection to imaging determines correlation with infarct core. AJNR Am J Neuroradiol 2012;33:E61, author reply E62

6. Brunner F, Tomandl B, Hanken $\mathrm{K}$, et al. Impact of collateral circulation on early outcome and risk of hemorrhagic complications after systemic thrombolysis. Int J Stroke 2012 Oct 23. [Epub ahead of print]

7. Campbell BC, Christensen S, Levi CR, et al. Cerebral blood flow is the optimal CT perfusion parameter for assessing infarct core. Stroke 2011;42:3435-40

8. Kamalian S, Konstas AA, Maas MB, et al. CT perfusion mean transit time maps optimally distinguish benign oligemia from true "at- risk" ischemic penumbra, but thresholds vary by postprocessing technique. AJNR Am J Neuroradiol 2012;33:545-49

9. Konstas AA, Goldmakher GV, Lee TY, et al. Theoretic basis and technical implementations of CT perfusion in acute ischemic stroke, Part 2. Technical implementations. AJNR Am J Neuroradiol 2009;30:885-92

10. Konstas AA, Goldmakher GV, Lee TY, et al. Theoretic basis and technical implementations of CT perfusion in acute ischemic stroke, Part 1. Theoretic basis. AJNR Am J Neuroradiol 2009;30:662-68

11. Sztriha LK, Manawadu D, Jarosz J, et al. Safety and clinical outcome of thrombolysis in ischaemic stroke using a perfusion CT mismatch between 3 and 6 hours. PloS One 2011;6:e25796

12. Obach V, Oleaga L, Urra X, et al. Multimodal CT-assisted thrombolysis in patients with acute stroke: a cohort study. Stroke 2011; 42:1129-31

13. Hesselmann V, Niederstadt T, Dziewas R, et al. Reperfusion by combined thrombolysis and mechanical thrombectomy in acute stroke: effect of collateralization, mismatch, and time to and grade of recanalization on clinical and tissue outcome. AJNR Am J Neuroradiol 2012;33:336-42

14. Turk A, Magarik JA, Chaudry I, et al. CT perfusion-guided patient selection for endovascular treatment of acute ischemic stroke is safe and effective. J Neurointerv Surg 2012;4:261-65

15. Amenta PS, Ali MS, Dumont AS, et al. Computed tomography perfusion-based selection of patients for endovascular recanalization. Neurosurg Focus 2011;30:E6

16. Albers GW, Thijs VN, Wechsler L, et al. Magnetic resonance imaging profiles predict clinical response to early reperfusion: the Diffusion and Perfusion Imaging Evaluation for Understanding Stroke Evolution (DEFUSE) study. Ann Neurol 2006;60:508-17

17. Davis SM, Donnan GA, Parsons MW, et al. Effects of alteplase beyond $3 \mathrm{~h}$ after stroke in the Echoplanar Imaging Thrombolytic Evaluation Trial (EPITHET): a placebo-controlled randomised trial. Lancet Neurol 2008;7:299-309

18. Butcher K, Parsons M, Allport L, et al. Rapid assessment of perfusion-diffusion mismatch. Stroke 2008;39:75-81

19. Furlan AJ, Eyding D, Albers GW, et al. Dose escalation of Desmoteplase for Acute Ischemic Stroke (DEDAS): evidence of safety and efficacy 3 to 9 hours after stroke onset. Stroke 2006;37:1227-31

20. Hacke W, Albers G, Al-Rawi Y, et al. The Desmoteplase in Acute Ischemic Stroke Trial (DIAS): a phase II MRI-based 9-hour window acute stroke thrombolysis trial with intravenous desmoteplase. Stroke 2005;36:66-73

21. Mishra NK, Albers GW, Davis SM, et al. Mismatch-based delayed thrombolysis: a meta-analysis. Stroke 2010;41:e25-33

22. Förster A, Gass A, Kern R, et al. MR imaging-guided intravenous thrombolysis in posterior cerebral artery stroke. AJNR Am J Neuroradiol 2011;32:419-21

23. Dani KA, Thomas RG, Chappell FM, et al. Computed tomography and magnetic resonance perfusion imaging in ischemic stroke: definitions and thresholds. Ann Neurol 2011;70:384-401

24. Zussman BM, Boghosian G, Gorniak RJ, et al. The relative effect of vendor variability in CT perfusion results: a method comparison study. AJNR Am J Neuroradiol 2011;197:468-73

25. Zussman B, Jabbour P, Talekar K, et al. Sources of variability in computed tomography perfusion: implications for acute stroke management. Neurosurg Focus 2011;30:E8

26. Zeger SL, Liang KY. Longitudinal data analysis using generalized linear models. Biometrics 1986;42:121-30

27. Katzman R, Clasen R, Klatzo I, et al. Report of joint committee for stroke resources. IV. Brain edema in stroke. Stroke 1977;8:512-40

28. http://www.jointcommission.org/certification/primary_stroke_centers. aspx Accessed October 31, 2013 